As a luminogram, coronary angiography provides a good overview of the coronary artery tree. Using quantitative coronary measurements, the degree of coronary obstruction can be determined. The limitation of coronary angiography is that it does not provide information on the arterial wall structure and therefore cannot assess the extent of atherosclerosis. Knowledge about adaptive coronary remodelling processes as compensatory enlargement of the coronary artery has focused diagnostic interest on the non-stenotic lesions of the coronary tree. Intravascular ultrasound (IVUS) can reveal discrepancies between the extent of coronary atherosclerosis and angiography imaging by in vivo plaque imaging. Spectrum analysis of IVUS-derived radiofrequency (RF) data enables a more detailed analysis of plaque composition and morphology. Preliminary in vitro studies correlated four histological plaque components with a specific spectrum analysis of the RF data. The different components (fibrous, fibrofatty, necrotic core and dense calcium) are colour coded. Coronary tissue maps were reconstructed from RF data using IVUS-Virtual Histology (VH IVUS) software (Real-Time VH, Volcano Corporation, Rancho Cordova, California, USA). VH IVUS has the potential to detect high-risk lesions and can provide new insights into the pathophysiology of coronary artery disease. VH IVUS allows the differentiation of different lesion types based on information derived from histopathology. The in vivo specific histological analysis of coronary atherosclerosis may allow better stratification of treatment of patients with coronary artery disease.

See end of article for authors' affiliations

Correspondence to: Dr A König, Department of Medicine, Division of Cardiology, Medizinische Poliklinik - Innenstadt, Ludwig - Maximilians Universität, Ziemssenstr 1, 80336 Munich, Germany; andreas.koenig@ med.uni-muenchen.de

Accepted 9 February 2007

Published Online First

13 May 2007

\section{HISTORY AND DEVELOPMENT OF THE TECHNOLOGY}

Intravascular ultrasound (IVUS) has been used since the 1990s as an additional tool for the examination of coronary arteries. Its ability to analyse vessel dimensions and pinpoint location of coronary plaques has been used for guidance of coronary interventions, ${ }^{1}$ analysis of ambiguous lesions especially, left main coronary artery disease $^{2}$ and clarification of the mechanism of instent restenosis and transplant vasculopathy. ${ }^{34}$ Owing to its comprehensive insight into periprocedural vessel dimensions, discrepancies between coronary angiography and IVUS imaging when assessing the extent and severity of coronary atherosclerosis were detected. ${ }^{5}$
Heart 2007;93:977-982. doi: 10.1136/hrt.2007.116384

IVUS is able to identify ruptured lesions, and recent studies showed that IVUS parameters indicated the risk for future plaque rupture. ${ }^{6}$ The use of IVUS in progression-regression trials provides the assessment of atheroma burden under specific medical influences such as statin therapy. ${ }^{7}$ IVUS imaging is limited for assessing the exact analysis of the plaque composition. Both calcified and dense fibrotic tissues have strong echo reflections with lateral shadowing and are, therefore, not easy to differentiate. The differential diagnoses of areas with low echo reflections include lipid-rich or fibrotic tissue, intraplaque haemorrhage or even thrombus and lumen area.

Because of the limitations of the greyscale analysis the importance of processing data from ultrasound backscattered RF-signals to determine the plaque composition was realised.

Spectral analysis of the RF signals provides a more detailed assessment of the plaque. Early studies with backscatter analysis showed a possible differentiation between different tissue types in vitro.

In recent studies, specific spectral parameters have been found to be indicative for certain tissue types after data aquisition with a $10 \mathrm{MHz}$ transducer and spectrum analysis with fast Fourier transformation. ${ }^{9}$ Studies using transducers with higher frequencies ${ }^{10}{ }^{11}$ showed that spectral analysis is able to discriminate between different tissue types.

Nair et $a l^{12}$ confirmed the superiority of autoregressive analysis over classic Fourier analysis in the determination of plaque composition. Four major plaque components of the coronary plaque have been identified to date (fibrous, fibrofatty, necrotic core and dense calcified tissue).

In this study, the ability of RF analysis to assess the plaque vulnerability was investigated.

\section{DESCRIPTION AND USE OF THE TECHNIQUE}

IVUS is an imaging technique enabling real-time high-resolution tomographic viewing of the coronary arteries. The morphology and distribution of the coronary plaques can be analysed. In addition, qualitative analysis and plaque characterisation is possible based on differing echogeneicity. Echo signals with poor reflection represent lipidic areas. Echo signals with strong reflection and lateral shadowing represent calcified areas. ${ }^{13}$

In traditional greyscale IVUS, the amplitude of the echo signal is used for interpretation of data difficult to analyse the different shades of grey

Abbreviations: ACS, acute coronary syndrome; RF, radiofrequency; TCFA, thin cap fibroatheroma 
(fig 1). Tissue characterisation is not provided. In addition to greyscale IVUS, the VH IVUS software (Volcano Corporation, Rancho Cordova, California, USA) enables automated border contour detection by estimation of the lumen and adventitial borders by spectral analysis. After planimetry, tissue classification is performed for the plaque area.

VH IVUS acquisition is performed during standardised IVUS pullback (Eagle Eye Gold catheter, Volcano Corporation; 3.5F/ $20 \mathrm{MHz}$ ) with use of VH IVUS console (Volcano Corporation). RF data are obtained for on-line greyscale IVUS imaging and VH IVUS imaging during the same pullback.

All IVUS catheters have variability in power output and signal-receiving sensitivity. Therefore, prior efforts at standardisation for border automation and tissue characterisation on greyscale IVUS were not successful. VH IVUS uses the raw RF signal and an elaborate automatic calibration technology (blind deconvolution) normalising for catheter-to-catheter and system-to-system variability allowing standardised image interpretation and tissue characterisation.

On the basis of ex vitro analysis, IVUS-derived RF analyses were compared with corresponding histopathological areas. The corresponding RF analyses were evaluated and the respective spectrum curves with spectrum parameters were calculated. Four major plaque components of the coronary plaque have been identified to date (fibrous, fibrofatty, necrotic core and dense calcified tissue; fig 2). The predictive accuracy for the specific plaque components is approximately $93.4 \%$ for fibrous and $94.6 \%$ for fibrofatty tissue, $95.1 \%$ for necrotic core and $96.8 \%$ for dense calcium. ${ }^{14}$

Fibrous tissue is identified as a dark green group of pixels on the VH IVUS screen. This tissue is characterised by bundles of collagen fibres with little or no lipid accumulation in the fibrous area and no evidence of macrophages representing inflammatory response. Fibrofatty tissue is identified as a light green group of pixels on the VH IVUS screen. This tissue is characterised by loosely packed collagen fibres with lipid accumulation. There is no necrotic tissue and no or few cholesterol clefts. Large areas of fibrofatty tissue retain matrix. Necrotic core tissue is identified as a red group of pixels on the VH IVUS screen. This tissue is characterised by a high level of lipid with many necrotic cells and remnants of dead lymphocytes and foam cells. There are few or no collagen fibres, the cellular structure (matrix) is not well organised and there is poor mechanical stability. Microcalcification is often present as a byproduct of dead cells, or as a result of intraplaque haemorrhage.

Dense calcium is identified as a white pixel group on the $\mathrm{VH}$ IVUS screen. This tissue is characterised by compact calcium crystals.

\section{CLINICAL ROLE OF THE TECHNOLOGY}

In recent studies, high-risk lesions and multiple plaque ruptures in acute patients were detected by IVUS, ${ }^{15}{ }^{16}$ even though IVUS imaging is limited regarding analysis of the exact plaque composition. Calcified and dense fibrotic tissues may have lateral shadowing and are therefore not easy to differentiate. The differential diagnoses of areas with low echo reflections includes lipid-rich or fibrotic tissue, intraplaque haemorrhage or even thrombus and lumen area. A more detailed diagnosis of the plaque composition is enabled by $\mathrm{VH}$ IVUS.

In vivo studies using VH IVUS have shown that vulnerable plaques (thin cap fibroatheromas, TCFAs) occur more often in patients with acute coronary syndrome (ACS) than in patients with stable angina. ${ }^{17}$ Angiographic studies detected that acute coronary occlusions leading to ST segment elevation myocardial infarction tend to cluster in predictable hot spots, especially in the proximal third of the coronary arteries ${ }^{18}$ VH IVUS analysis confirmed these findings. Depending on the distance from the coronary ostium, the proximal segments showed significantly higher levels of necrotic core, but no change in the resting plaque components. ${ }^{19}$

Positive remodelling processes increasing plaque proliferation were shown $^{20}$ and confirmed by IVUS. ${ }^{21}$ VH IVUS analysis confirmed the relationship of outward and inward remodelling processes to plaque composition. In a small study, the in vivo plaque composition and morphology assessed using IVUS-RF analysis was related to coronary remodelling, supporting the role of plaque composition in the mechanisms of vessel remodelling. Positive remodelling is associated with high-risk lesions such as fibroatheromas or TCFA, whereas negative remodelling is associated with less vulnerable lesions such as intimal thickening or fibrous plaque composition. ${ }^{22}$

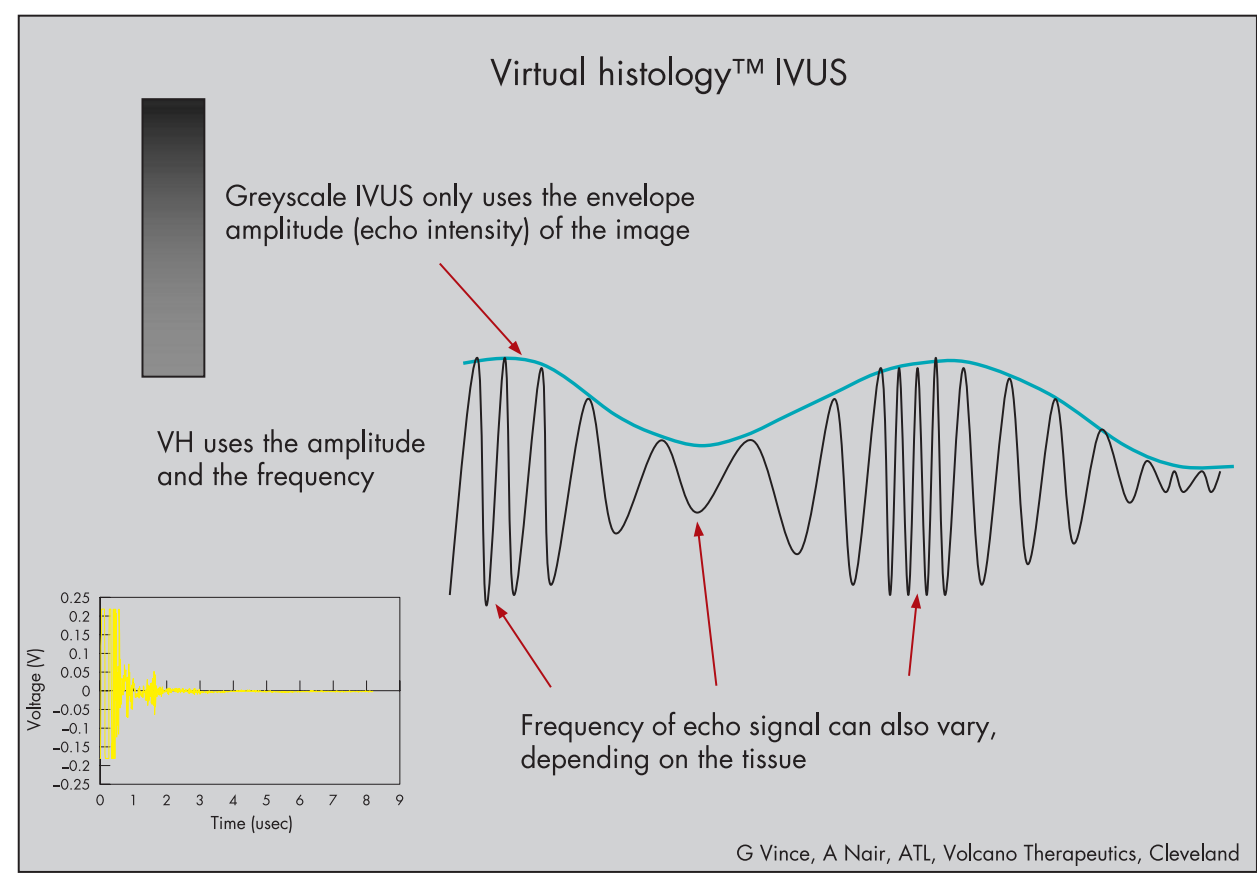

Figure 1 The radiofrequency signal received by the intravascular ultrasound (IVUS) catheter is divided into frequency "windows". The signal profile from each of those windows is processed through sophisticated algorithms. The signal profile is then matched to a known database of signal profiles previously mapped to one of the four main tissue types found in coronary plaques by histology. The decision tree assigns the corresponding colour to the orientationmatched pixel on the image. Finally, the virtual histology $(\mathrm{VH})$ image is reconstructed. 

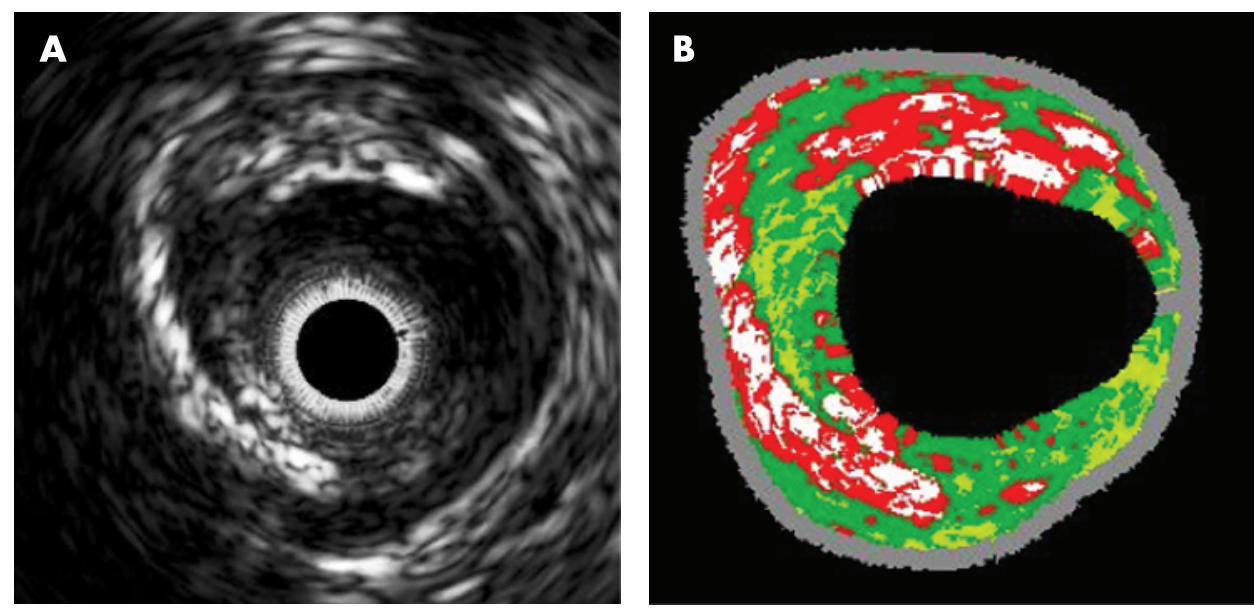

Figure 2 Automatic characterisation of the atherosclerotic plaque by virtual histology. After comparison of the raw frequency dataderived spectrum curves with the spectrum parameter of the database, the four colourcoded plaque components produced are presented. (A) Intravascular ultrasoundgreyscale image showing large excentric plaque morphology. (B) The corresponding colour-coded virtual histology image depicts the specific plaque components.

In terms of secondary prevention, it is clinically important to detect progression of coronary artery disease and, moreover, to predict coronary lesions with significant progression up to ACS. Angiographic studies showed clinically relevant progression in non-culprit lesions with a non-target lesion percutaneous coronary intervention during l year follow-up, significantly depending on the degree of coronary artery disease. ${ }^{23}$ Earlier angiographic studies showed different types of stenosis progression in terms of mild and rapid progression. ${ }^{24}$ Complex lesion morphology and unstable presentation were important factors for rapid stenosis progression. ${ }^{25}$ However, coronary angiography and clinical parameters were poor surrogates to predict future events in a broad cohort of patients who had percutaneous coronary intervention. In addition, non-invasive imaging failed to identify coronary plaques with potential rupture and consecutive ACS. This highlights the need for further study to identify potentially vulnerable lesions.

Catheter-based invasive diagnosis with VH IVUS can distinguish between different plaque types. It is able to detect criteria of plaque vulnerability. Criteria for increased vulnerability based on histopathological studies are, at present, the extent of confluent necrotic core, evidence of fibrotic cap, pattern of calcification, positive coronary remodelling, degree of luminal stenosis and localisation of the interrogated lesion.

However, there is currently no evidence to favour invasive treatment of a vulnerable plaque. A recent meta-analysis found the re-stenosis risk after revascularisation in angiographic intermediate lesions corresponded with the re-stenosis risk in clinically relevant stenoses and was therefore unacceptably high. ${ }^{26}$

We need prospective randomised studies to evaluate prophylactic treatment with coronary stent implantation in comparison with medical treatment. The PROSPECT (Providing Regional Observations to Study Predictors of Events in the Coronary Tree) trial is a natural history study to analyse the relationship of unexpected acute coronary syndrome and progression of coronary artery disease. This is the first prospective study to detect lesions that are considered prone to rupture with intravascular imaging.
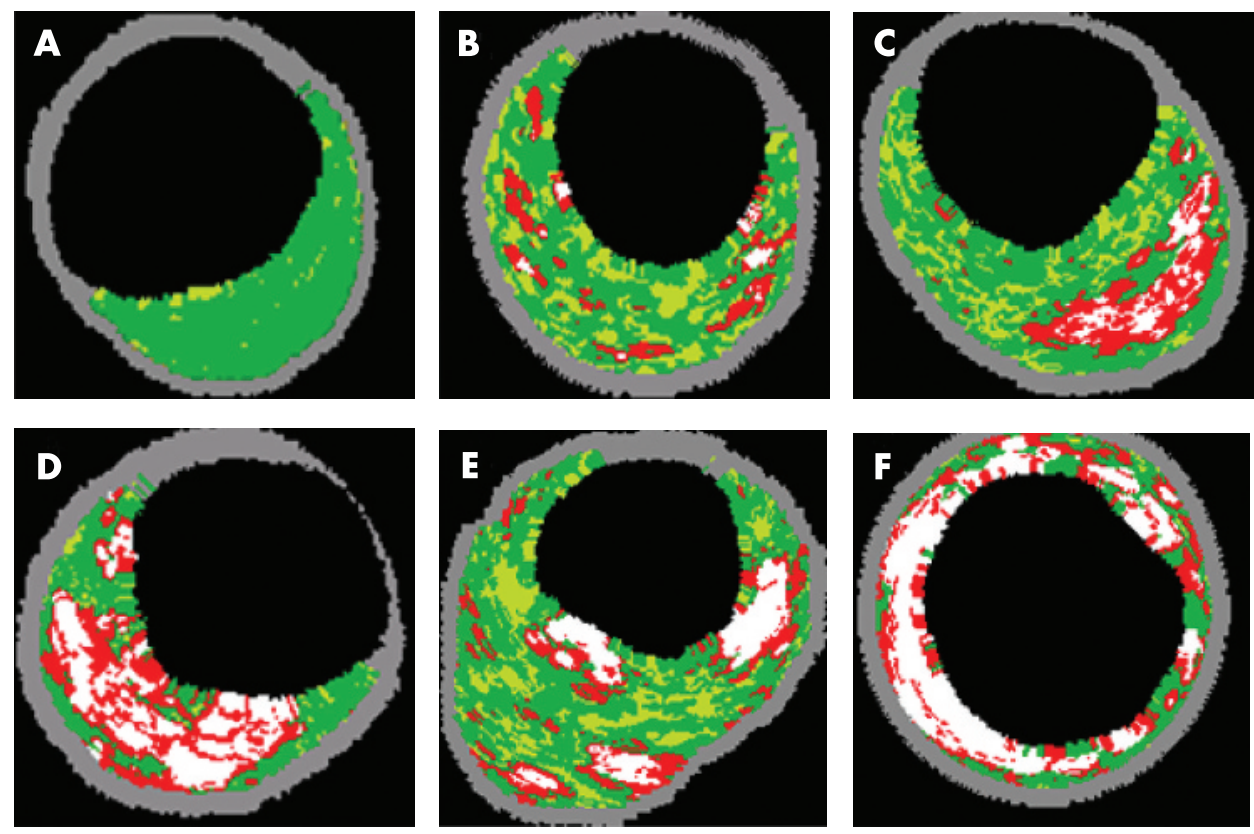

Figure 3 Plaque classification by intravascular ultrasound-virtual histology distinguishes between intimal thickening $(A, B)$, and more vulnerable lesions, such as fibroatheroma (C, D, E). In thin cap fibroatheroma (TCFA) (D), the necrotic core is lying on the surface of the plaque. Compared with the fibroatheroma (C) the fibrous cap is not visible. TCFA with multiple layers of necrotic areas (E) suggests multiple previous ruptures. (A) Adaptive intimal thickening; (B) pathological intimal thickening; (C) fibroatheroma; (D) IVUSdefined TCFA; (E) TCFA, multiple layer; (F) fibrocalcific plaque. 
The longitudinal analysis of a target lesion detects the extent of the necrotic core and the vulnerable plaque. As a consequence, treatment of the lesion should cover this plaque and potentially avoid future ruptures instead of covering just the minimum lumen site.

In vivo plaque classification by VH IVUS is based on histopathological studies. ${ }^{27}$ Coronary lesions are differentiated in plaques with adaptive and pathological intimal thickening, fibroatheromas and fibrotic calcified plaques. For risk stratification, it is important to differentiate between the aforementioned plaque types, and especially to distinguish between adaptive intimal thickening and fibrous cap atheroma (fig 3).

"Niche" interventional applications of greyscale IVUS are the characterisation and quantitative analysis of left main coronary artery disease, transplant vasculopathy and planning of debulking strategies. The additional information about the exact plaque composition by VH IVUS in these clinical settings may further optimise stratification of these patients.

As IVUS is best able to assess stent underexpansion and malapposition, IVUS guidance may play an increasing role in the drug-eluting stent era. In addition to IVUS-guided quantitative stent optimisation, determination of the plaque composition and distribution by VH IVUS may be helpful for choosing stent types and dimensions and therefore may further improve the clinical outcome.

\section{CURRENT LIMITATIONS OF THE TECHNIQUE}

A current limitation is the automatic border detection, which necessitates correction of the planimetry. Accurate borders are critically important, as the VH software characterises the entire plaque area with the four tissue types. Accurate border correction affords experience in greyscale IVUS imaging and analysis. Longer segments of plaque can be analysed, but this is time consuming and therefore not practical in the catheterisation laboratory. Whole segment analysis is available for off-line assessment.

The axial resolution (100-200 $\mu \mathrm{m})$ is too low to detect critical fibrous cap thickness, which is currently defined as $65 \mu \mathrm{m}$; however the threshold for critical cap thickness is under consideration and is probably higher. The introduction of the next generation high-frequency IVUS catheter enables better axial resolution.
Despite better differentiation of low echogenic reflexes with VH IVUS, differential diagnosis between soft plaque material and thrombus is currently not possible by RF analysis. Thrombus detection could help localise the extent and also origin of the plaque rupture in patients with ACS. The probability of detecting the tissue components in the plaque area correctly is very high. In our opinion, this accuracy is weakened in areas of lateral shadowing due to calcified or dense fibrotic tissue. This may influence the exact measurement of the amount and extent of necrotic core. As the area crucial for vulnerability is near the surface of the plaque, this limitation does not directly account for the underestimation of vulnerable lesions.

The VH IVUS analysis represents, in terms of an analysed TCFA, the current vulnerability of the interrogated lesion at that stage. The duration of this vulnerable stage and the exact time interval of progression or regression of coronary artery disease are unknown. Thrombus, as the primary surrogate for acute coronary thrombosis cannot be detected to date and therefore has to be excluded from the VH IVUS analysis.

Keeping in mind that the vulnerability of high-risk lesions may be only temporary, with changing plaque structures in short time intervals, prospective serial VH IVUS studies should be performed in vivo to clarify the natural history of these vulnerable lesions.

\section{FUTURE DEVELOPMENT}

VH IVUS is the most promising technique to detect vulnerable plaques and therefore to assess their natural history. Prospective trials using serial VH IVUS analysis may help to distinguish plaques prone to rupture and, as a consequence, to lead to cardiac events.

The treatment of these vulnerable lesions and coronary artery disease might be changed according to the results of these prospective studies. Regarding the technical development, the next high-frequency IVUS catheter (Revolution, $45 \mathrm{MHz}$, Volcano Corporation) is a rotational IVUS imaging catheter enabling increased spatial resolution in grayscale IVUS and accordingly VH IVUS. The phased-array and rotational catheters can be used on the same platform.

A cross-sectional area analysis and also length analysis will provide a vessel profile imaging in addition to the tomographic

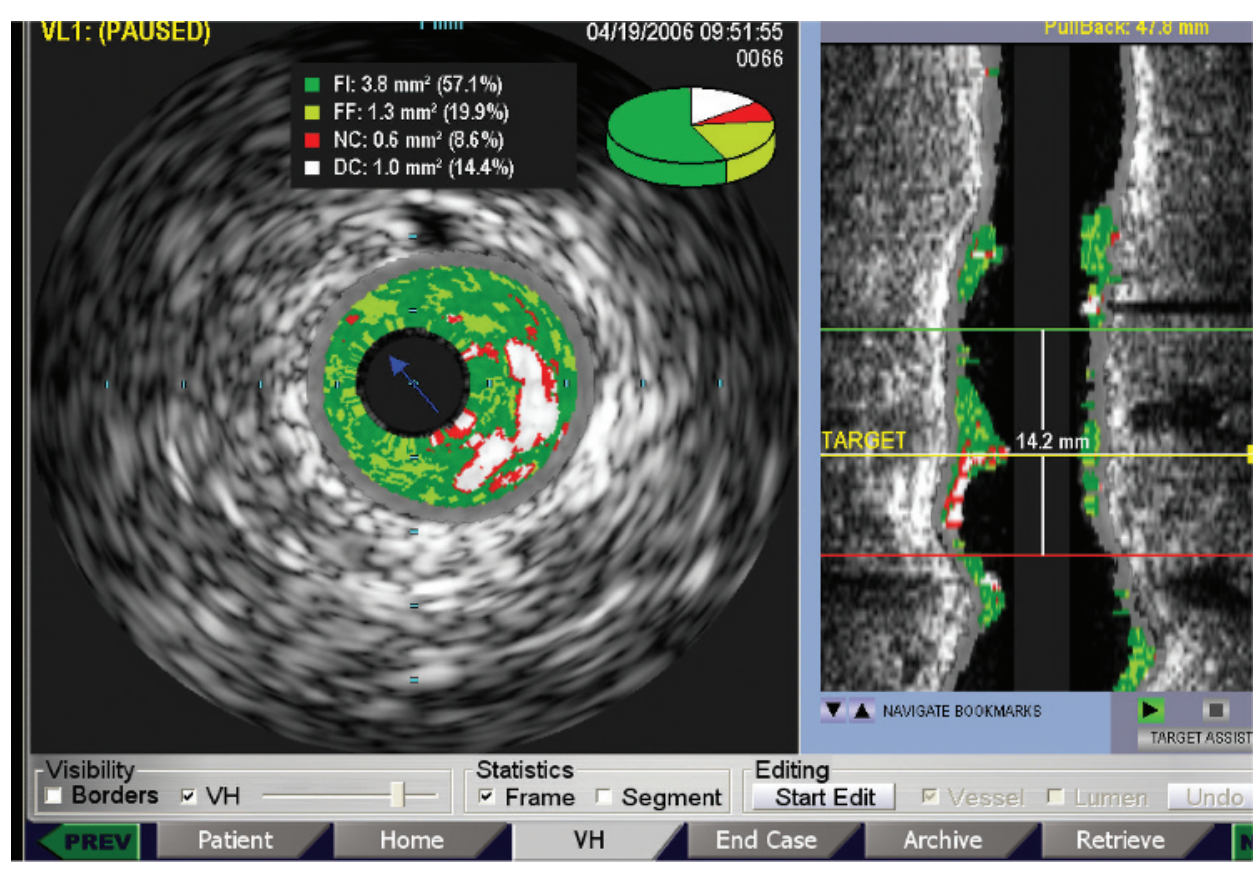

Figure 4 Volcano s5/i provides on-line real-time intravascular ultrasound-virtual histology in both tomographic and longitudinal views during image acquisition. 


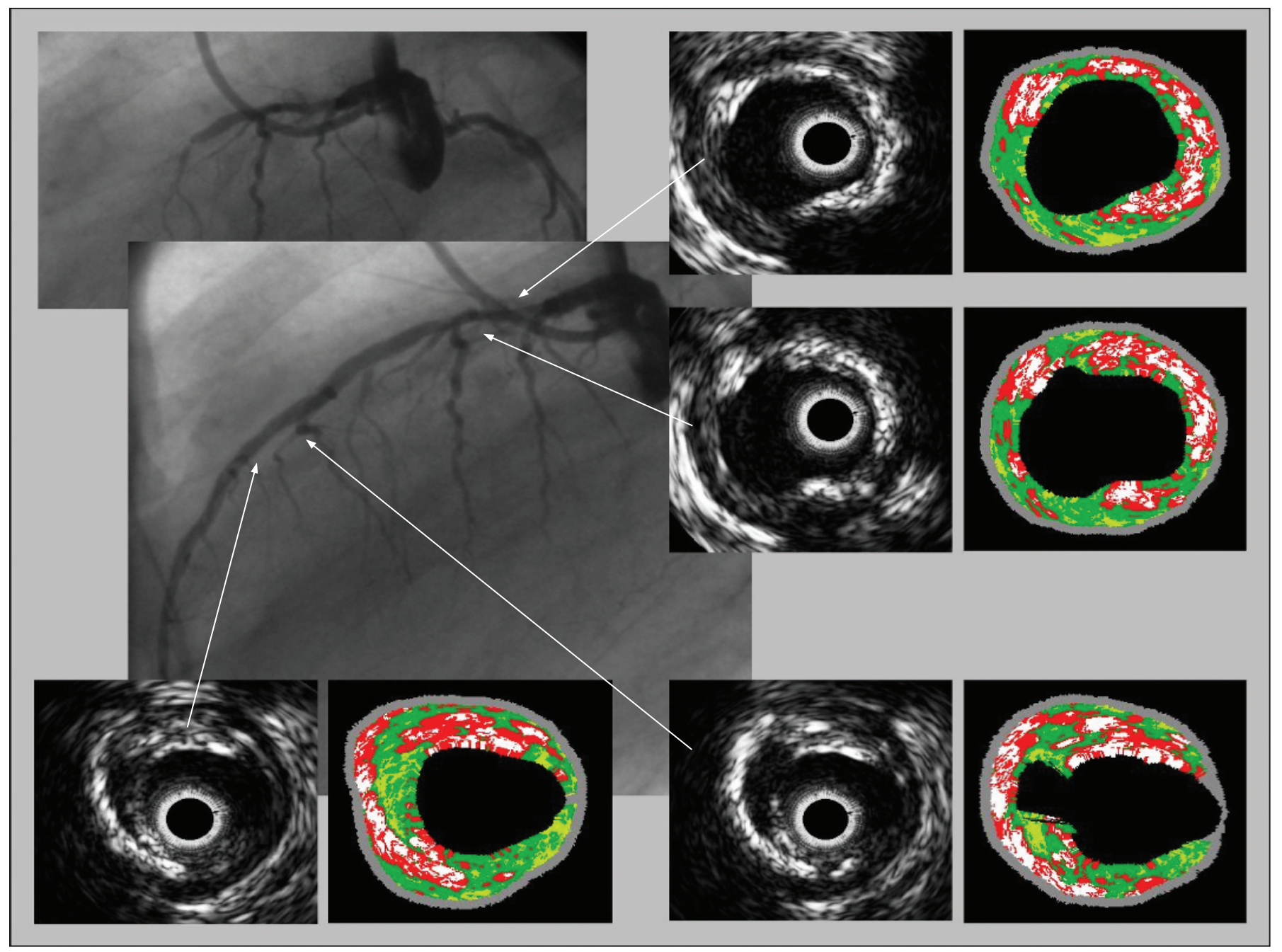

Figure 5 Patient with an acute coronary syndrome and occluded left anterior descending artery. Greyscale intravascular ultrasound shows severe atherosclerosis in the entire vessel and plaque rupture in the medial segment. The corresponding VH images show a high level of necrotic core and several layers of necrotic core with microcalcification, suggesting previous plaque ruptures.

view. Quantitative and qualitative lesion length analyses are provided to view the extent and distribution of the necrotic area. The incorporation of IVUS in the catheterisation laboratory will be optimised. The Volcano s5/i IVUS imaging system allows for customised incorporation of IVUS into the interventional catheterisation laboratory. In addition, Volcano s5/i provides true real-time VH IVUS overlaid onto the greyscale cross section and onto the longitudinal view during image acquisition (fig 4). AIM (Angio IVUS Mapping) is a future option with synchronised coregistration of VH IVUS and coronary angiography (collaboration with Paieon Medical, Rosh Haaiyn, Israel) providing a mapping of IVUS images to two- or three-dimensional quantitative coronary angiography.

\section{CONCLUSION}

Coronary angiography and clinical parameters are poor surrogates to predict future events in patients with coronary artery disease. This highlights the need for further studies to identify potentially vulnerable lesions. VH IVUS is currently the best catheter-based imaging tool to detect most of the criteria of plaque vulnerability based on pathology. Detection of high-risk lesions may have an influence on the treatment and prevention of acute coronary syndrome (fig 5). In clinical practice, several issues can be addressed by VH IVUS. On the basis of classification of pathological lesions, fibroatheroma can be distinguished from lesions with intimal thickening, and vulnerability criteria allow further risk stratification of fibrous cap atheroma. Currently, we have no evidence to support treatment of vulnerable lesions as a preventive strategy because there is uncertainty regarding the re-stenosis risk compared with the spontaneous rupture rate of high-risk lesions. Our knowledge about the natural history of atherosclerosis including lesion classification is mainly based on histopathological studies. As VH IVUS enables in vivo diagnostic of atherosclerotic histopathology, we can complete our understanding of the genesis and progression of this disease, avoiding selective patient cohorts.

Using VH-derived lesion analysis, coronary interventions may be modified using lesion-specific strategies. With the knowledge of the longitudinal distribution of vulnerable lesions, the culprit lesion is easier to identify and better treated by complete stent coverage.

The additional value of VH IVUS in specially indicated IVUSguided coronary interventions (niche IVUS application) needs to be determined.

\section{Authors' affiliations}

Andreas König, Volker Klauss, Department of Medicine, Division of Cardiology, Medizinische Poliklinik - Innenstadt, Munich, Germany

Competing interests: None declared. 


\section{REFERENCES}

1 Colombo A, Hall P, Nakamura S, et al. Intracoronary stenting without anticoagulation accomplished with intravascular ultrasound guidance. Circulation 1995:91:1678-88.

2 Abizaid AS, Mintz GS, Abizaid A, et al. One year follow-up after intravascular ultrasound assessment of moderate left main coronary artery disease in patients with ambiguous angiograms. J Am Coll Cardiol 1999;34:707-15.

3 Mintz GS, Popma JJ, Hong MK. et al, Intravascular ultrasound to discern devicespecific effects and mechanisms of restenosis.Am J Cardiol, 1996;78:18-22.

4 Klauss V, Ackermann K, Spes $\mathrm{CH}$, et al. Coronary plaque morphologic characteristics early and late after heart transplantation: in vivo analysis with intravascular ultrasonography. Am Heart J 1997;133:29-35.

5 Nissen SE, Yock P. Intravascular ultrasound: Novel pathophysiological insights and current clinical applications. Circulation 2001;103:604-16.

6 Yamagishi M, Terashima M, Awano K, et al. Morphology of vulnerable plaque: insights from follow-up of patients examined by intravascular ultrasound before an acute coronary syndrome. J Am Coll Cardiol 2000;35:106-11.

7 Nissen SE. Application of intravascular ultrasound to characterize coronary artery disease and assess the progression or regression of atherosclerosis. Am J Cardiol 2002:89:24B-31B.

8 Wilson LS, Neale ML, Talhami HE, et al. Preliminary results from attenuationslope mapping of plaque using intravascular ultrasound. Ultrasound Med Biol 1994;20:529-42.

9 Lizzi FL, Greenebaum M, Feleppa EJ, et al. Theoretical framework for spectrum analysis in ultrasonic tissue characterization. J Acoust Soc Am 1983:73:1366-73.

10 Spencer T, Ramo MP, Salter Dm, et al. Characterisation of atherosclerotic plaque by spectral analysis of $30 \mathrm{MHz}$ intravascular ultrasound radio frequency data. Proc IEEE Ultrason Sympos 1996;2:1073-6.

11 Moore MP, Spencer T, Salter DM, et al. Characterisation of coronary atherosclerotic morphology by spectral analysis of radiofrequency signal: in vitro intravascular ultrasound study with histological and radiological validation. Heart 1998:79:459-67.

12 Nair A, Kuban BD, Tuzcu EM, et al. Coronary plaque classification with intravascular ultrasound radiofrequency data analysis. Circulation 2002; 106:2200-6

13 Potkin BN, Bartorelli AL, Gessert JM, et al. Coronary artery imaging with intravascular high-frequency ultrasound. Circulation 1990;81:1575-85.
14 Nair A, Margolis MP, Kuban BD, et al. Automated coronary plaque characterisation with intravascular ultrasound backscatter: ex vivo validation. Eurolntervention 2007;3:113-20.

15 Rioufol G, Finet G, Ginon I, et al. Multiple atherosclerotic plaque rupture in acute coronary syndrome: a three-vessel intravascular ultrasound study. Circulation 2002;106:804-8

16 Tanaka A, Shimada K, Sano T, et al. Multiple plaque rupture and c-reactive protein in acute myocardial infarction. J Am Coll Cardiol 2005;45:1594-9.

17 Rodriguez-Granillo GA, Garcia-Garcia HM, Mc Fadden EP, et al. In vivo intravascular ultrasound-derived thin cap fibroatheroma detection using ultrasound radiofrequency data analysis. J Am Coll Cardiol 2005;46:2038-42.

18 Wang JC, Normand SLT, Mauri L, et al. Coronary artery spatial distribution of acute myocardial infarction occlusions. Circulation 2004;110:278-84.

19 Valgimigli M, Rodriguez-Granillo GA, Garcia-Garcia HM, et al. Distance from the ostium as an independent determinant of coronary plaque composition in vivo: an intravascular ultrasound study based radiofrequency data analysis in humans. Eur Heart J 2006;27:655-63.

20 Glagov S, Weinberg E, Zarins CK, et al. Compensatory enlargement of human atherosclerotic coronary arteries. N Engl J Med 1987;316:1371-5.

21 Schoenhagen $\mathbf{P}$, Ziada KM, Kapadia SR, et al. Extent and direction of arterial remodeling in stable vs. unstable coronary syndromes. An intravascular ultrasound study. Circulation 2000;101:598-603.

22 Rodriguez-Granillo GA, Serruys PW, Garcia-Garcia HM, et al. Coronary artery remodelling is related to plaque composition. Heart 2006:92:388-91.

23 Glaser R, Selzer F, Faxon DP, et al. Clinical progression of incideal, asymptomatic lesions discovered during culprit vessel coronary intervention. Circulation 2005;111:143-9.

24 Yokoya K, Takatsu H, Suzuki T, et al. Process of progression of coronary artery lesion from mild or moderate stenosis to moderate or severe stenosis. A study based on four coronary arteriograms per year. Circulation 1999;100:903-9.

25 Kaski JC, Chester MR, Chen L, et al. Rapid angiographic progression of coronary artery disease in patients with angina pectoris. The role of complex stenosis morphology. Circulation 1995;92:2058-65.

26 Mercado N, Maier W, Boersma E, et al. Clinical and angiographic outcome of patients with mild coronary lesions treated with balloon angioplasty or coronary stenting. Implications for mechanical plaque sealing. Eur Heart J 2003;24:541-51.

27 Virmani R, Kolodgie FD, Burke AP, et al. Lessons from sudden coronary death: comprehensive morphological classification scheme for atherosclerotic lesions. Arterioscler Thromb Vasc Biol 2000;20:1262-75.

\section{Stay a step ahead with Online First}

We publish all our original articles online before they appear in a print issue. This means that the latest clinical research papers go straight from acceptance to your browser, keeping you at the cutting edge of medicine. We update the site weekly so that it remains as topical as possible. Follow the Online First link on the home page and read the latest research. 